\title{
Characterization of the Au Atomic Contact in a Hydrogen Environment Using Vibration Spectroscopy of a Single Molecular Junction*
}

\author{
Manabu Kiguchi ${ }^{\dagger}$ \\ Division of Chemistry, Graduate School of Science, Hokkaido University, \\ Kita 10, Nishi 8, Sapporo, 060-0810, Japan and PRESTO, \\ Japan Science and Technology Agency, Sapporo, 060-0810, Japan
}

Tomoka Nakazumi, Kunio Hashimoto, and Kei Murakoshi Division of Chemistry, Graduate School of Science, Hokkaido University, Kita 10, Nishi 8, Sapporo, 060-0810, Japan (Received 10 November 2008; Accepted 25 December 2008; Published 31 January 2009)

\begin{abstract}
We have developed a mechanically controllable break junction (MCBJ) system in ultra high vacuum (UHV). The $\mathrm{Au}$ atomic contact in a hydrogen environment was investigated using this newly developed system. The conductance of the $\mathrm{Au}$ atomic contact decreased with the introduction of hydrogen gas. The vibration spectra showed symmetric peaks around $\pm 50 \mathrm{meV}$, which could be assigned to the vibration mode between Au and hydrogen. The conductance and vibration spectroscopy measurement suggested that the hydrogen atom or molecule would adsorb on or incorporate into the Au atomic contact. [DOI: 10.1380/ejssnt.2009.53]
\end{abstract}

Keywords: Electrical transport measurements; Gold; Hydrogen molecule; Nano-wires

\section{INTRODUCTION}

Building electronic devices using individual molecules is one of the ultimate goals in nanotechnology. To achieve this end, a variety of single molecular junctions have been fabricated using many techniques, such as scanning tunneling microscopy, electron-beam lithography, focused ion-beam lithography, and mechanically controllable break junction (MCBJ) [1]. The single molecular junction has not been well characterized since, in most cases, only its conductance has been investigated. Thus, it is desirable to characterize the single molecular junction using spectroscopic measurements. Vibration spectroscopy of a single molecular junction is a promising method with which to characterize the single molecular junction $[2-7]$. The vibration energy provides information on the single molecular junction, such as chemical species, adsorption site and orientation of the molecule.

The vibration spectroscopy of a single molecular junction has been applied to single hydrogen, benzene, oxygen, $\mathrm{C}_{60}$, and alkanedithiol molecular junctions [2-7]. In the case of the $\mathrm{Pt}$ atomic contact in a hydrogen environment, simultaneous conductance and vibration spectroscopy measurements revealed the formation of the $\mathrm{Pt}$ atomic chain in contact with a single hydrogen molecular bridge [3]. While some single molecular junctions have been characterized using vibration spectroscopy of a single molecular junction, this technique has only been applied to limited cases. Therefore, the fundamental mechanism of the vibration spectroscopy of a single molecular junction has not been completely clear up to now.

In the present study, we have tried to fabricate the newly developed MCBJ system to measure the vibration spectrum of a single molecular junction. As a represen-

\footnotetext{
* This paper was presented at International Symposium on Surface Science and Nanotechnology (ISSS-5), Waseda University, Japan, 9-13 November, 2008.

$\dagger$ Corresponding author: kiguti@sci.hokudai.ac.jp
}

tative single molecular junction, we focused on the single hydrogen molecule bridging between Au electrodes. Recently, the conductance behavior of the Au atomic contact was investigated in the hydrogen environment. The conductance of the Au atomic contact decreased with the introduction of hydrogen gas, suggesting the formation of the single hydrogen molecular junction [8]. Here, it should be noted that hydrogen does not adsorb on the surface of bulk Au crystal [9]. Although the formation of the single hydrogen molecular junction was suggested by the conductance measurement, the structure of the junction was not clear. In the present study, we have investigated the $\mathrm{Au}$ atomic contact in a hydrogen environment using the newly developed MCBJ system.

\section{EXPERIMENTAL}

Figure 1 shows a schematic view and picture of the MCBJ system used to measure the vibration spectrum of a single molecular junction. The metal nano contact and single molecular junction were fabricated using the MCBJ technique (see Fig. 1(d)) [10, 11]. The bending substrate was a plate of phosphor bronze about $1 \mathrm{~mm}$ thick, $20 \mathrm{~mm}$ long and $8 \mathrm{~mm}$ wide. The surface of the plate was insulated with a thin polymer foil (Kapton). The center of the $0.1 \mathrm{~mm}$ diameter Au wire was notched by a surgical knife to create a weak spot. Then, the wire was glued onto the bending beam with two drops of epoxy adhesive (Stycast $2850 \mathrm{FT}$ ). The distance between the drops was manually reduced under a microscope. The whole assembly was mounted on a three-point bending mechanism, consisting of a stacked piezo-element (PI: P-249K007) and two fixed counter supports. By bending the substrate, the top surface of the substrate was expanded, and the wire was elongated. At some critical strain, the wire finally broke, forming two separated electrodes. By relaxing the bending of the substrate, the contact could be re-established.

The MCBJ part was set at the end part of the insert. Figure 1(c) shows the end of the insert. The vacuum pot was attached to the bottom part of the insert to create a 

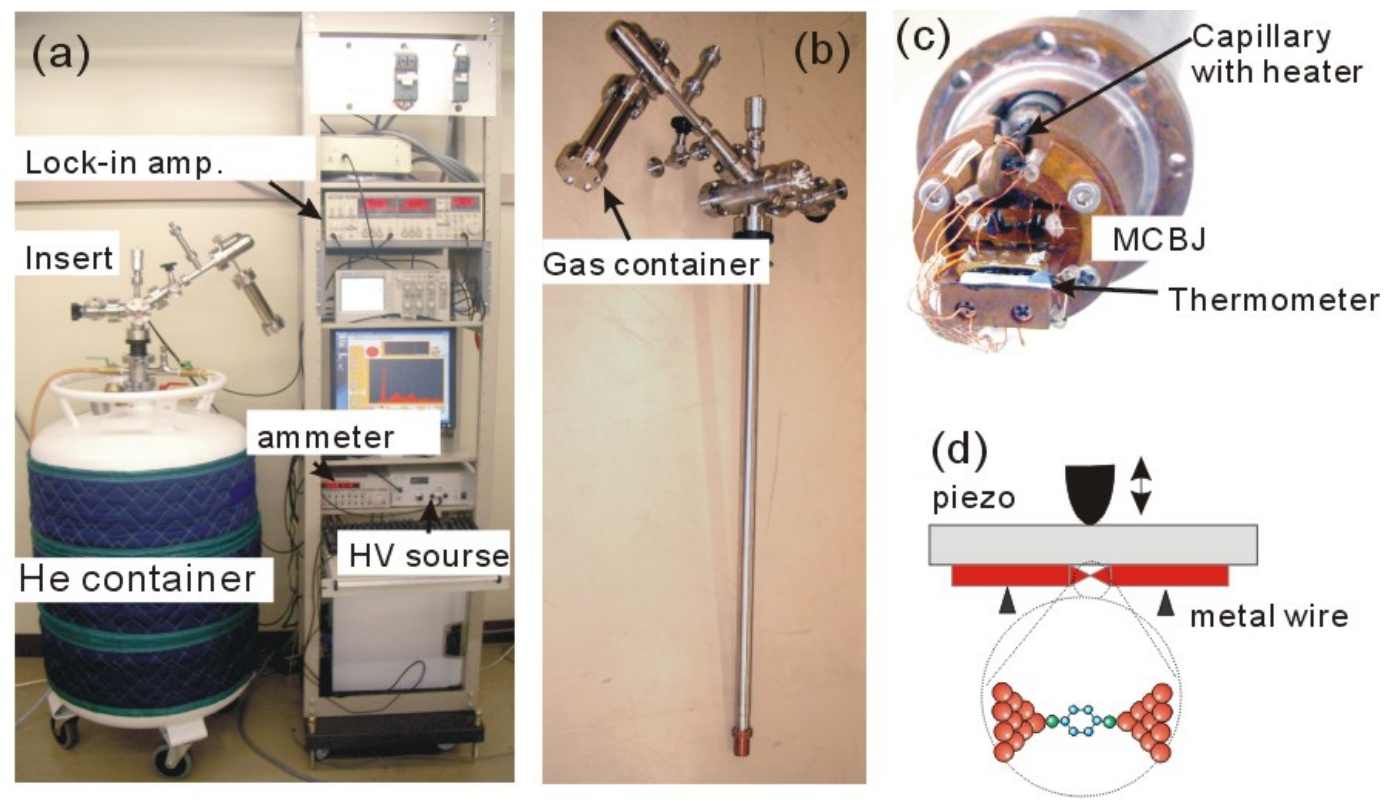

FIG. 1: (a) Side view of the measurement system for the vibration spectroscopy of a single molecular junction, (b) side view of the insert, (c) picture of the end part of the insert, and (d) schematic side view of MCBJ.

vacuum chamber (Fig. 1(b)). After evacuating the insert, the insert was put into the liquid He container to cool the sample (Fig. 1(a)). The temperature of the sample was around $10 \mathrm{~K}$. Hydrogen gas was introduced to the metal nano contact via a home made capillary equipped with a heater wire. The vibration spectrum was measured using a standard lock-in technique. The conductance was recorded for the fixed contact configuration using an $\mathrm{AC}$ modulation of $1 \mathrm{mV}$ amplitude and a frequency of 7.777 $\mathrm{kHz}$, while slowly ramping the DC bias between -100 and $+100 \mathrm{mV}$.

\section{RESULTS AND DISCUSSION}

Figure 2(a) shows the typical conductance trace of the $\mathrm{Au}$ nano contacts in UHV at $10 \mathrm{~K}$. The conductance of the Au nano contacts decreased in a stepwise fash-
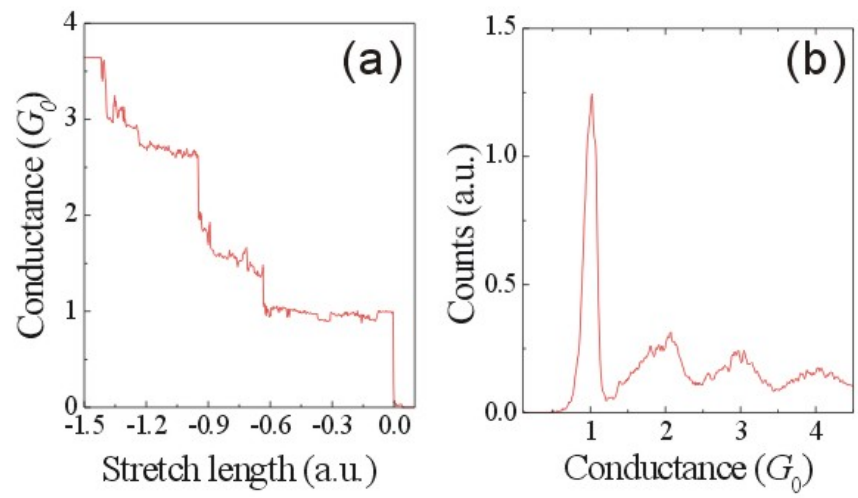

FIG. 2: (a) Conductance trace and (b) conductance histogram of the Au nano contacts at $10 \mathrm{~K}$. ion with each step occurring at integer multiples of $G_{0}$ $\left(G_{0}=2 e^{2} / h\right)$. The corresponding conductance histogram (Fig. 2(b)) showed well-defined peaks around 1, 2, and $3 G_{0}$. The observed conductance quantization behavior agreed with the previous results studied in UHV, air [10] and solution [12, 13], indicating that the conductance quantization behavior of metal nano contacts could be successfully observed with our newly developed system.

Next, the Au atomic contact in a hydrogen environment was investigated with this setup. Figure 3 shows the conductance histogram of the $\mathrm{Au}$ nano contacts after introduction of hydrogen gas. The bias voltage was changed from 20 to $200 \mathrm{mV}$. At the bias voltage of 20 $\mathrm{mV}$, the conductance trace showed steps after showing step at $1 G_{0}$ (see inset of Fig. 3(a)). The conductance values of the steps below $1 G_{0}$ varied with the conductance traces. The corresponding conductance histogram showed a broad feature below $1 G_{0}$ and a large background above $1 G_{0}$. The appearance of steps and features below $1 G_{0}$ agreed with previous studies [8]. The intensity of the features below $1 G_{0}$ gradually decreased and disappeared at a bias voltage of above $200 \mathrm{mV}$. The change in the conductance histogram was continuous and reversible.

The conductance behavior indicated that the structure showing the conductance value below $1 G_{0}$ was unstable at a high bias voltage. At the high bias voltage, the temperature of the contact increased by the joule heating, and, thus, the interaction between hydrogen and the Au atomic contact was weakened at the high bias voltage [16]. It is noteworthy that the conductance feature below $1 G_{0}$ appeared at a low bias voltage, even after it first disappeared at a high bias voltage. This indicated that the hydrogen was not blown off the surface but rather remained bound to one of the two electrodes at the high bias voltage.

Next, the origin of the conductance feature below $1 G_{0}$ is discussed. The appearance of the feature below $1 G_{0}$ suggested some interaction between the hydrogen and the $\mathrm{Au}$ 

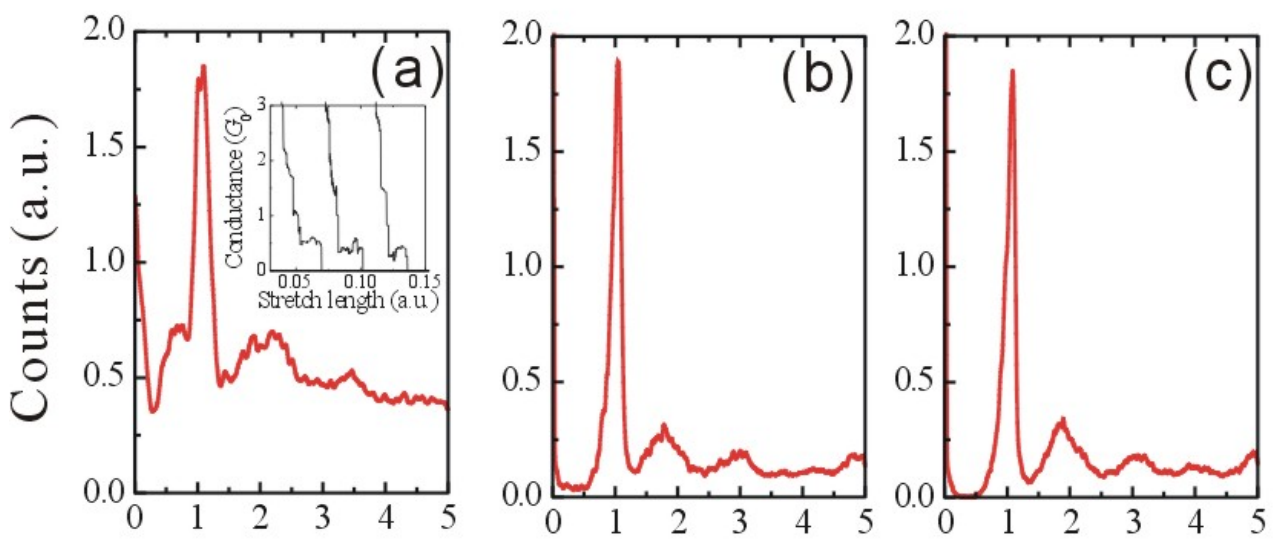

Conductance $\left(G_{0}\right)$

FIG. 3: Conductance histograms of the Au nano contacts in a hydrogen environment at the bias voltages of (a) $20 \mathrm{mV}$, (b) 100 $\mathrm{mV}$, and (c) $200 \mathrm{mV}$. Inset shows the conductance trace of the $\mathrm{Au}$ nano contact in a hydrogen environment at the bias voltage of $20 \mathrm{mV}$.

atomic contact. The interaction between the Au atomic contact and hydrogen molecule or atom has been investigated by theoretical calculations. Barnett et al. showed that the conductance of the $\mathrm{Au}$ atomic contact decreased to $0.6-0.01 G_{0}$ with hydrogen incorporation into the $\mathrm{Au}$ atomic contact, possibly due to scattering or interference of conducting electrons in the contact [14]. Jelinek et al. showed that the conductance decreased to $0.7-0.5 G_{0}$ with hydrogen adsorption onto the contact [15]. The experimental results and theoretical calculation results suggest that the hydrogen atom or molecule could adsorb onto or incorporate into the $\mathrm{Au}$ atomic contact when hydrogen gas was introduced into the $\mathrm{Au}$ atomic contact.

The hydrogen atom or molecule also affected the structure and conductance of the $\mathrm{Au}$ nano contacts other than the $\mathrm{Au}$ atomic contact. The conductance histogram showed the large background above $1 G_{0}$ at a bias voltage of $20 \mathrm{mV}$ (see Fig. 3(a)). The large background originates from the increase in counts and the broadening of the peaks. The increase in counts indicates the stabilization of the $\mathrm{Au}$ nano contacts. In solution, $\mathrm{Au}, \mathrm{Fe}$, $\mathrm{Co}$, and $\mathrm{Ni}$ nano contacts were stabilized by molecular adsorption onto the nano contacts [17]. This stabilization was explained by the decrease in the surface energy of the nano contact caused by the molecular adsorption onto them. Therefore, the stabilization of the Au nano contact could be explained by the decrease in the surface energy which was induced by the hydrogen adsorption onto the Au nano contact. The broadening of the peaks could be also explained by the hydrogen adsorption onto or incorporation into the Au nano contact. The electronic structure of the metal nano contact, which defines the conductance, depends on the atomic configuration of the metal nano contact [10]. In the present experimental conditions, hydrogen adsorption or hydrogen incorporation into the structure of the Au nano contact could not be well defined. Therefore, the Au nano contact would not show a fixed conductance value, leading to the broadening of the peaks in the conductance histogram.

The interaction between the Au atomic contact and hy- drogen was suggested by the conductance measurements as discussed in the previous section. However, there is no direct evidence of this interaction. In order to get the evidence of this interaction, the vibration spectroscopy of a single molecular junction was applied to the contact showing conductance below $1 G_{0}$. Figure 4 shows an example of the differential conductance $(d I / d V)$ and its derivative $\left(d I^{2} / d V^{2}\right)$ for the Au atomic contact taken in a hydrogen environment at a conductance of about $0.1 G_{0}$. The spectrum showed an increase in the differential conductance symmetrically around $50 \mathrm{meV}$, and clear symmetric peaks were observed in the second derivative $\left(d I^{2} / d V^{2}\right)$. The symmetric feature around 30 50 meV could be observed for various contacts showing conductance of $0.01-0.7 G_{0}$

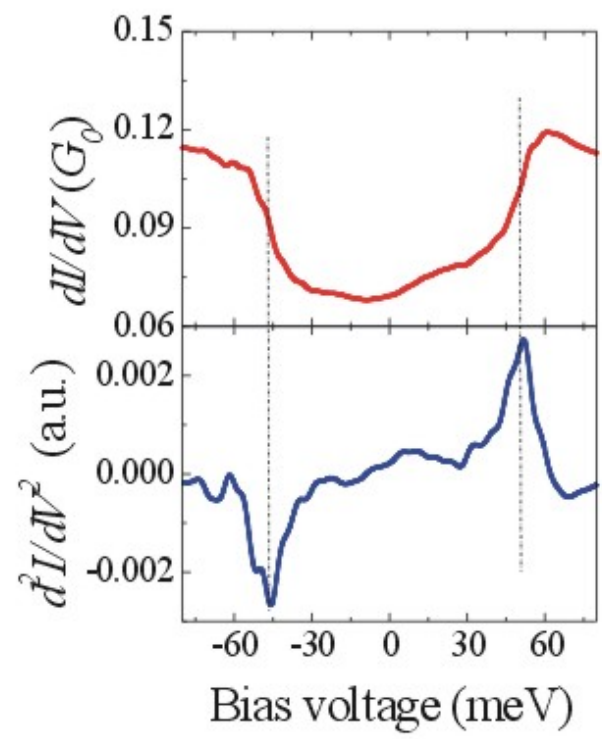

FIG. 4: Conductance and derivative for the Au atomic contact in a hydrogen environment at $10 \mathrm{~K}$. 
in more than 5 independent samples. The increase in the conductance and peaks in the second derivative could be explained by the phonon excitation at the single molecular junction [7]. When the bias voltage was increased and crossed the threshold for excitation of a vibration mode, an additional tunneling channel for electrons was opened. This opening of the new channel resulted in a sudden increase in the differential conductance at the threshold voltage, which is observed as peaks in the second derivative. Since the phonon energy of $\mathrm{Au}$ is around 10-20 meV [18], the observed peaks in the spectra would originate from the vibration mode between $\mathrm{Au}$ and hydrogen. We might succeed in observing evidence of the interaction between hydrogen and Au using vibration spectroscopy of single molecular junction. At this moment, it is not clear whether hydrogen is adsorbed onto the Au atomic contact or whether hydrogen is incorporated into the contact. Further investigation and theoretical calculation are needed to determine the atomic configuration showing conductance below $1 G_{0}$.

\section{CONCLUSIONS}

In conclusion, we have investigated the Au atomic contact in a hydrogen environment with the newly developed
MCBJ system. The conductance of the Au atomic contact decreased with the introduction of hydrogen gas. The vibration spectra of the contact with the conductance below $1 G_{0}$ showed symmetric peaks around $\pm 50 \mathrm{meV}$, which could be assigned to the vibration mode between the $\mathrm{Au}$ atomic contact and hydrogen.

\section{Acknowledgments}

We would like to express our sincere gratitude to Prof. J. M. van Ruitenbeek at Leiden University for the many stimulating discussions and Mr. T. Kato at Hokkaido University for his technical support in preparing the IETS measurement system. This work was supported by a Grant-in-Aid for Scientific Research on Priority Areas "Electron transport through a linked molecule in nanoscale" (No. 17069001) from MEXT.
[1] N. J. Tao, Nature Nanotechnol. 1, 173 (2006).

[2] R. H. M. Smit, Y. Noat, C. Untiedt, N. D. Lang, M. C. van Hemert and J. M. van Ruitenbeek, Nature 419, 906 (2002).

[3] M. Kiguchi, R. Stadler, I. S. Kristensen, D. Djukic, and J. M. van Ruitenbeek, Phys. Rev. Lett. 98, 146802 (2007).

[4] M. Kiguchi, O. Tal, S. Wohlthat, F. Pauly, M. Krieger, D. Djukic, J. C. Cuevas, and J. M. van Ruitenbeek, Phys. Rev. Lett. 101, 046801 (2008)

[5] W. H. A. Thijssen, M. Strange, J. M. J. aan de Rrugh, and J. M. van Ruitenbeek, New J. Phys. 10, 33005 (2008).

[6] T. Bohler, A. Edtbauer, and E. Scheer, Phys. Rev. B 76, 125432 (2007).

[7] J. Hihath, C. R. Arroyo, G. Rubio-Bollinger, N. Tao, and N. Agrait, Nano Lett. 8, 1673 (2008).

[8] Sz. Csonka, A. Halbritter, and G. Mihaly, Phys. Rev. B. 73, 075405 (2006).

[9] A. Zangwill, Physics at Surfaces (Cambridge University Press, 1998).
[10] N. Agrait, A. L. Yeyati, and J. M. van Ruitenbeek, Phys. Rep. 377, 81 (2003).

[11] M. Kiguchi and K. Murakoshi, J. Phys. Chem. C 112, 8140 (2008).

[12] M. Kiguchi, T. Konishi, and K. Murakoshi, Phys. Rev. B 73, 125406 (2006).

[13] M. Kiguchi, T. Konishi, K. Hasegawa, S. Shidara, and K. Murakoshi, Phys. Rev. B 77, 245421 (2008).

[14] R. N. Barnett, H. Hakkinen, A. G. Scherbakov, and U. Landman, Nano Lett. 4, 1845 (2004).

[15] P. Jelinek, R. Perez, J. Ortega, and F. Flores, Phys. Rev. Lett. 96, 046803 (2006).

[16] Y. Chen, M. Zwolak, and M. Ventra, Nano Lett. 3, 1691 (2003).

[17] H. X. He, C. Shu, C. Z. Li, and N. J. Tao, J. Electroanal. Chem. 522, 26 (2002).

[18] N. Agrait, C. Untiedt, G. Rubio-Bollinger, and S. Vieira, Phys. Rev. Lett. 88, 216803 (2002). 